\title{
Simulation of a Matrix Converter Fed Drive with Sliding Mode Control
}

\author{
Jan Bauer \\ Dept. of Electric Drives and Traction, Czech Technical University in Prague, Faculty of Electrical Engineering, Technická 2, \\ 16627 Praha, Czech Republic
}

Corresponding author: bauerja2@fel.cvut.cz

\begin{abstract}
Induction machines are among the most widely used electrical-to-mechanical converters in electric drives. Their advantageous robustness and simplicity goes hand-in-hand with complicated control. A converter with a suitable control algorithm is needed in order to withdraw maximum power and dynamics from the drive. In recent times, control methods such as those based on DTC and sliding mode methods have come to the forefront, due to their robustness and relative simplicity.

In the field of power converters, new converter topologies are emerging with improved efficiency that pushes the operation limits of the drive. This paper focuses on the development of a control of this kind of strategy for an induction machine fed from a matrix converter.
\end{abstract}

Keywords: matrix converter, induction machine model, sliding mode control.

\section{Introduction}

The field of regulated AC drives is very broad [6, 10], but most of the drives are based on induction machines and frequency converters. Indirect frequency converters are still the most widely used type, but they have several disadvantages. In order to push the limits of IM drives and achieve maximum efficiency, research in this field is also investigating other converter topologies, e.g. multilevel inverters [10] and so-called all-silicon solutions [8]. Matrix converters have also been attracting some interest.

The matrix converter [3] does not include a passive accumulation element in DC link, and uses nine bidirectional switches to transfer voltage from its input to the output. It has some attractive features in comparison with indirect frequency converters:

- The output frequency is almost unlimited. The only limit is the maximum switching frequency of the devices that are used.

- It has a relatively simple power circuit. An indirect frequency converter comprises a rectifier, an inverter, and a DC link with a passive accumulation element. A matrix converter has only nine bidirectional switches. They are mostly realized from discreet semiconductor modules, but several manufacturers already offer integrated compact IGBT modules.

- The features of the matrix converter are same as VSI, with an active front end on its input. However, there is a drawback: because of the absence of a DC link, the output voltage amplitude is

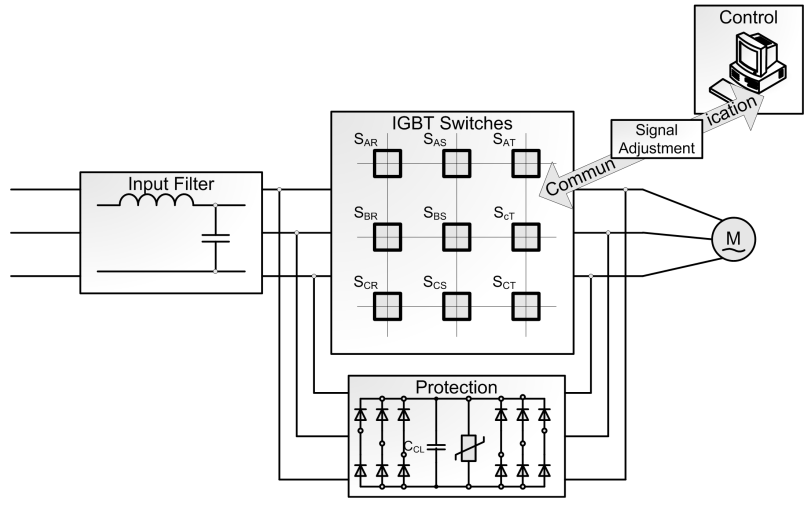

Figure 1: Matrix converter.

limited to $86.6 \%$ of the input voltage amplitude if we wish to maintain sinusoidal input currents.

- Sinusoidal input and output currents.

- Power factor regulation.

- Work in all four quadrants.

The matrix converter got its name because its switches can be formed into a two-dimensional matrix. Topology, consisting of nine bidirectional switches, is depicted in Fig. 1.

The modulation strategy and the control algorithm are very important parts of the converter's controller. Widely used modulation strategies from the area of indirect frequency converters are generally not applicable because of the absence of a DC link. Modulation strategies based on virtual DC-link space vector modulation were therefore developed $[2,5,4]$. This approach helps to reduce the modulation complexity, and enables classical modulation strategies to be 
reused (after a slight modification). At the same time, the approach works as a dependency injection. Various approaches can therefore be used to control the output voltage and the input current at the same time. This paper presents phase control of the input current in combination with sliding mode control of the output voltage. This enables us to overcome the traditional limitation of the output voltage without abandoning input current control.

As concerns the control algorithm, Field-Oriented Control (FOC) and the Direct Torque Control (DTC) are the most widely used high performance induction motor control methods at the present time [1]. In FOC, torque and flux are controlled through decoupled control of the stator current: the torque and flux-producing components are controlled separately. The current is controlled by fast inner control loops. The outputs of the current controllers are often led to a decoupler, where the coupling between the torque and flux producing current components is compensated. In addition, since it operates in field coordinates, it requires coordinate transformation between the stationary and rotating flux coordinates.

All this makes FOC a relatively complicated control method that puts high demands on precise generation of the reference voltage in order to provide a good current control. In DTC, however, the torque and flux are controlled directly by switching suitable voltage vectors. This is relatively close to sliding mode based control.

Let us turn to the sliding mode $[7,9]$. In the case of many scientific topics, ideas and approaches, it is difficult to say exactly where a particular theory originated. Some of the first attempts can be found in the work of Irmgard Lotz. She developed many ideas and applied them already in the Second World War. Her results were heavily reworked and improved in the former Soviet Union by Stanislav V. Emelyanov, and especially, later, by Vadim I. Utkin. Nowadays, sliding mode theory is popular all over the world. There are numerous publication about it, and this control approach is applied in many areas of engineering.

Sliding mode theory originated from the systems that naturally included a kind of relay and in where its usage often cannot be omitted. In systems of this kind, classical theories are often unsatisfactory. However, there are also many areas and systems that do not include any kind of relay, and yet sliding mode control can be successfully implemented and used to provide robustness and a well-defined response to big changes in the target value of the controlled parameter. Power electronics is a scientific field in which nonlinearity and switching is essential, due to technical circumstances that do not allow power switches to work in linear mode. Power electronics and drives are therefore very suitable fields for using sliding mode control.

In most cases, sliding mode is based on guiding the system state space vector exactly via suitable switching following the sliding surface. However, the state vector first has to reach the switching plane, and the state space vector will only then move more or less directly to the destination position. This approach can invoke a time delay when the controller is starting up, but it can be implemented easily using a simple analog comparator - i.e. using analog equipment. It can be proved whether this approach is stable for a particular task, and what its limits are.

Control action based on a change in target value can be divided into three main steps or phases:

1. drive system to a stable manifold (the reaching phase);

2. slide to equilibrium (the sliding phase);

3. maintain the target value (the maintenance phase).

Classical sliding mode control has its strong point in the sliding phase, where it is accompanied by robustness. A weak point is the initial reaching phase, and there is sometimes a problem of chatter in the maintenance phase. In real systems, this is caused by limited switching frequency, and by the particular set of real available or natural switching states.

The induction machine is a fourth-order system, and there are two action inputs that are, when supplied from voltage source inverter, bundled into eight vectors with a predefined direction and value. We only select a vector among this set of vectors. The possible directions of the vector depend on the construction of the inverter. The amplitude is given by the DC link voltage, and it cannot be changed quickly or cannot be changed at all (for a matrix converter, a change can be made in a virtual rectifier by adding a switching step). The switching frequency is limited to some hundreds of $\mathrm{Hz}$ (for high-power drives) or to tens of $\mathrm{kHz}$ (for smaller drives). The target point does not depend on the value of the state variables, but on multiplication of the state variables (mechanical torque). We might attempt to define the criteria for the best control law as follows: The aim is to move from any current system state (defined by the system state variables) to any target system state in the shortest possible time. This can be simplified to the task of finding the shortest route to the target point. That is currently defined by a line segment. There is a $1 \mathrm{D}$ space of system states that deliver the same mechanical torque value for the electric motor. All of them are good candidates for the target state of the control algorithm.

Existence and stability can be evaluated by analyzing the state space description of the induction motor in order to find limit boundaries, where the available action vectors can no longer produce the required movement in all directions. In other words, the 
boundary can be defined as the system state where the time derivative of one particular state variable is equal to zero, and at the same time the other available vectors deliver the same sign (the state value achieves its limit, and it can no longer be increased).

By applying this line segment approach, we may be able to eliminate the sliding mode reaching phase, and if necessary we may obtain comparable performance to the sliding phase for a machine that is not yet excited, e.g. typically a machine that is just starting. Of course, in the maintenance phase, both the classical sliding mode and the line segment approach may produce unwanted chatter. This can be overcome by additional arrangements, but this topic lies beyond the scope of this paper.

\section{Model of the matrix converter}

Since modulation is not the main topic of this paper, a simplified model is fully sufficient [3]. Let us use the virtual dc-link concept (Fig. 2). Under this consideration, the matrix converter can be virtually divided into a virtual rectifier and a virtual inverter. This will enable the application of classical modulation approaches, even in a direct converter.

The inverter part can then be imagined as three bi-state switches. This means either $+U_{\mathrm{DC}}$ or $-U_{\mathrm{DC}}$ can appear on the output terminals. By contrast, a virtual rectifier can be imagined as two tri-state switches [2]. This guarantees the possibility that the direction of the input current space vector can be controlled independently from the selected output voltage vector.

Based on the power balance, and assuming zero losses in the converter, the transfer function of the converter can be defined as

$$
u_{\mathrm{out}}=m_{U} m_{I} u_{\mathrm{in}} .
$$

When we employ space vector theory on the virtual rectifier part, we obtain formulas determining the duty ratio of the input current and output voltage vectors. Equation

$$
\left(\begin{array}{c}
d_{i y} \\
d_{i \delta}
\end{array}\right)=\frac{\sqrt{6}}{3} \frac{\left|i_{\mathrm{in}}\right|}{i_{\mathrm{P} \alpha}}\left(\begin{array}{c}
\sin \left(\frac{\pi}{3}-\theta_{i}\right) \\
\sin \theta_{i}
\end{array}\right)
$$

represents the input current. The situation is depicted in the Fig. 3 left.

This switching will cause the converter to consume almost sinusoidal current, and the virtual DC-link voltage will be constant.

The situation in the virtual inverter part will be different, since in our case one of the six space vectors will be used without any additional modulation (the
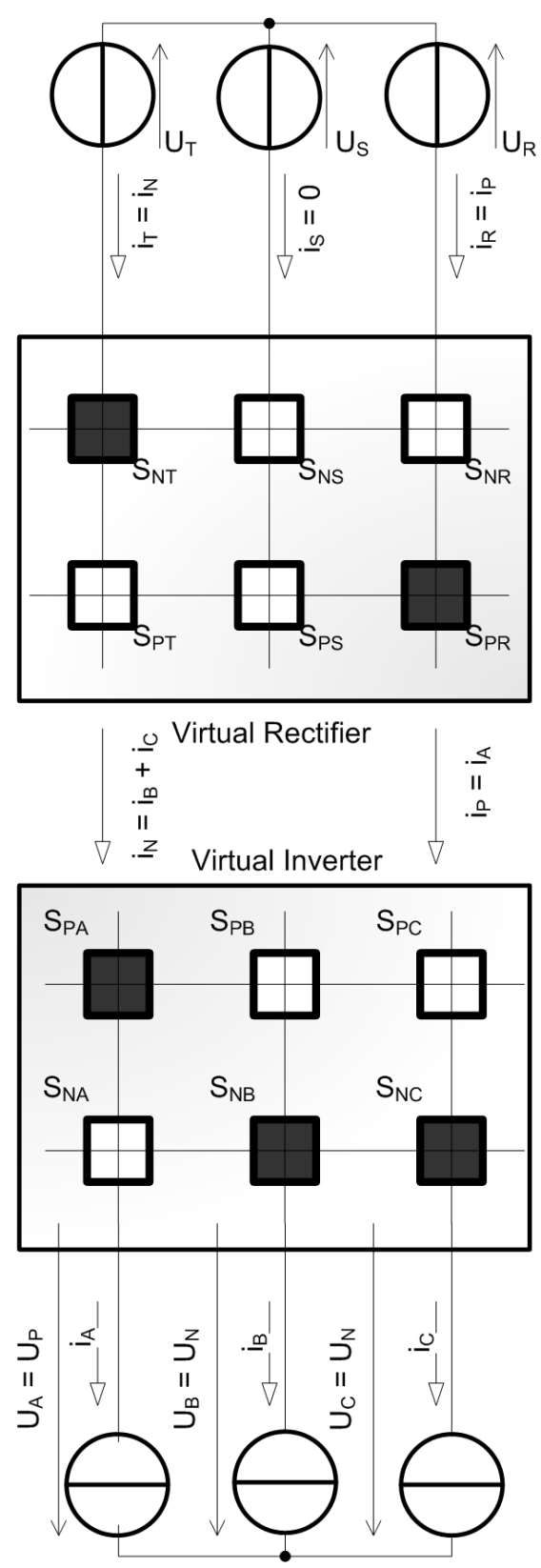

Figure 2: Virtual DC-link.
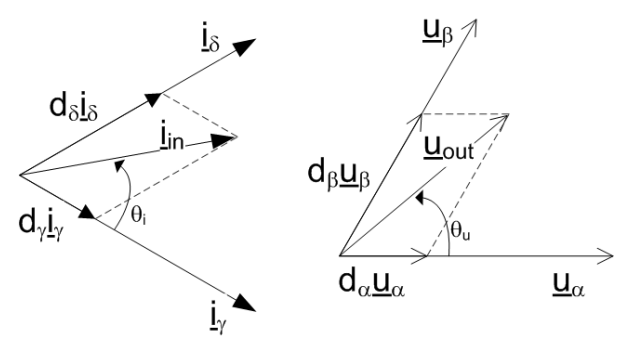

Figure 3: Synthesis of the input current vector and output voltage vector. 

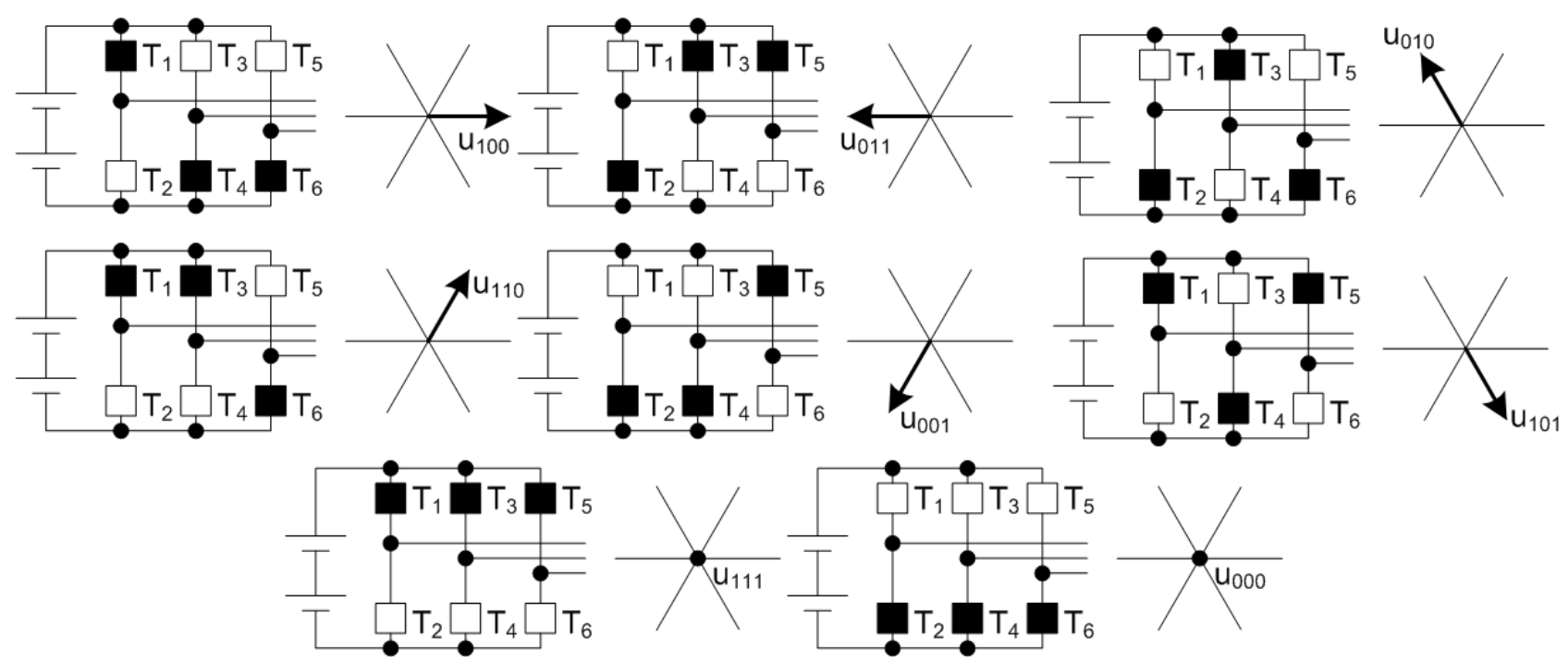

Figure 4: Virtual inverter output vectors.

output modulation will be a product of the sliding mode control).

Independently from the input sinusoidal modulation, there is a free choice among the vectors in Fig. 4.

Because the output voltage will be switched without any modulation, its amplitude corresponds to the amplitude of the voltage in the virtual DC link. Therefore, the only way to influence its amplitude is by modulating the input current. The current modulation index can be expressed assuming a power balance in the converter:

$$
\begin{aligned}
P_{\mathrm{DC}} & =P_{\text {in }} \\
u_{\mathrm{DC}} i_{\mathrm{DC}} & =\left|u_{\text {in }}\right|\left|i_{\text {in }}\right| \cos \varphi .
\end{aligned}
$$

For the modulation index of the virtual inverter (Fig. 3 right) we can write

$$
m_{U}=\frac{\frac{\left|u_{\mathrm{out}}\right|}{k}}{\frac{\sqrt{3}}{2} u_{\mathrm{DC}}}=\frac{3}{2} \frac{\left|u_{\mathrm{out}}\right|}{u_{\mathrm{DC}}} \frac{2}{\sqrt{3}}=\sqrt{3} \frac{\left|u_{\mathrm{out}}\right|}{u_{\mathrm{DC}}},
$$

where $k=2 / 3$ is a transformation coefficient that ensures power invariant transformation.

The input modulation index of the virtual rectifier can be expressed with the help of Fig. 3 as

$$
m_{I}=\frac{\frac{\left|i_{\mathrm{in}}\right|}{k}}{\frac{\sqrt{3}}{2} \sqrt{3} i_{\mathrm{DC}}}=\frac{\left|i_{\mathrm{in}}\right|}{i_{\mathrm{DC}}} .
$$

When we substitute (5) and (6) into (4), the final equation for calculating the input current modulation index $m_{I}$ can be formed:

$$
m_{I}=\sqrt{3} \frac{\left|u_{\mathrm{out}}\right|}{\left|u_{\mathrm{in}}\right|} .
$$

From the equations of the machine, we can easily calculate the voltage that is required to hold the machine in the desired stable state $\left(u_{o} u t\right)$ and the input voltage is also known.

\section{Model of an asynchronous machine}

In order to obtain maximum performance from the drive, a precise regulation algorithm is needed. These algorithms are mostly based on regulating the flux of the machine, which can scarcely be measured. Machine parameters from equivalent circuit and measured values are used to estimate the inner machine flux based on machine equations. The final accuracy of the equivalent circuit, and thereby also the accuracy of the controller, depends on exact knowledge of the equivalent circuit values. A further important advantage of the equivalent circuit is that the behavior of the machine, including the stable operation area, the maximum achievable torque, etc., can be derived analytically from the mathematical description of the equivalent circuit. This will be performed below. Finally, the control algorithm for the electrical torque of the machine will be designed on the basis of this knowledge.

The $\Gamma$-equivalent circuit (Fig. 5) will be used in this paper to describe the behavior of the machine. The advantage of this circuit is the simplification due to fusing the rotor and stator inductances into a single inductance on the rotor side, without loss of information.

The induction machine is a system with four state variables. The following equation describes derivation of both fluxes according to state matrix $A$ and vector of inputs $b$ :

$$
\Psi^{\&}(t)=b(t)+A \Psi(t) .
$$

Any combination of fluxes or currents can be used. The behavior of the machine can be derived analytically from (8). However, since information about the rotor flux cannot be obtained easily, only stator 


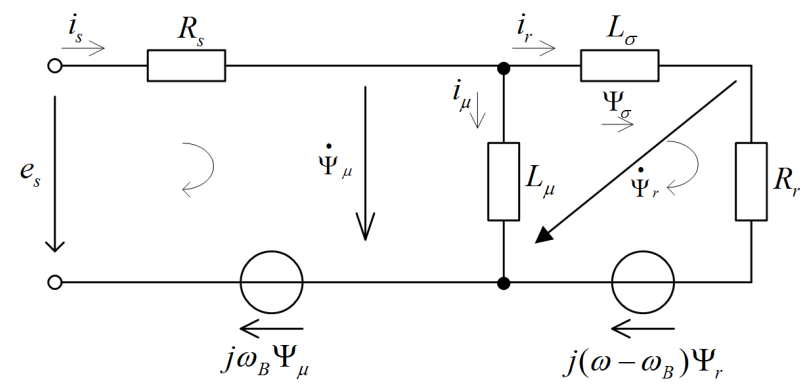

Figure 5: Equivalent circuit of IM.

variables are used in this paper for modeling IM. The system based on stator variables taken from inside the $\Gamma$-equivalent circuit is introduced in:

$$
\left(\begin{array}{c}
\Psi_{\mu}^{\&}(t) \\
i_{\mathrm{s}}^{\&}(t)
\end{array}\right)=A_{\mathrm{s}}\left(\begin{array}{c}
\Psi_{\mu}(t) \\
i_{\mathrm{S}}(t)
\end{array}\right)+b_{\mathrm{s}}(t),
$$

where $A_{\mathrm{s}}$ is the main state space matrix, $b$ represents the influence of action vectors, and are given by:

$$
\begin{gathered}
A_{\mathrm{s}}=\left(\begin{array}{cccc}
0 & \omega_{\mathrm{B}} & -R_{\mathrm{S}} & 0 \\
-\omega_{\mathrm{B}} & 0 & 0 & -R_{\mathrm{S}} \\
\sigma_{2} & \frac{\omega \sigma_{3}}{L_{\mu} L_{\sigma}} \sigma_{1} & \omega_{\mathrm{B}}-\omega & \\
-\frac{\omega \sigma_{3}}{L_{\mu} L_{\sigma}} & \omega_{2} & \omega-\omega_{\mathrm{B}} & -\sigma_{1}
\end{array}\right) \\
\text { where } \sigma_{1}=-\frac{R_{r} L_{\mu}+R_{\mathrm{s}} L_{\mu}+R_{\mathrm{s}} L_{\sigma}}{L_{\mu} L_{\sigma}}, \\
\sigma_{2}=\frac{R_{r}}{L_{\mu} L_{\sigma}} \\
\text { and } \sigma_{3}=L_{\mu}+L_{\sigma} ; \\
b_{\mathrm{s}}(t)=\left(\begin{array}{c}
e_{\mathrm{sa}}(t) \\
e_{\mathrm{sb}}(t) \\
\frac{e_{\mathrm{sa}}(t) L_{\mu}+e_{\mathrm{sa}}(t) L_{\sigma}}{L_{\mu} L_{\sigma}} \\
\frac{e_{\mathrm{sb}}(t) L_{\mu}+e_{\mathrm{sb}}(t) L_{\sigma}}{L_{\mu} L_{\sigma}}
\end{array}\right) .
\end{gathered}
$$

The last important missing equation is the expression for the calculating of the torque of the induction machine:

$$
M_{E}=\frac{3}{2} p(\Psi \times i)=\frac{3}{2}\left(\Psi_{\mathrm{d}} i_{\mathrm{q}}-\Psi_{\mathrm{q}} i_{\mathrm{d}}\right) .
$$

We can propose the first simplifying assumption that the coordinate system rotates with speed of the flux. Therefore $\omega_{\mathrm{B}}=\omega_{1}, \Psi_{\mathrm{d}}=\Psi$ and $\Psi_{\mathrm{q}}=0$. Using this dynamically rotating coordinate system the

\begin{tabular}{|c|c|c|c|}
\hline \multicolumn{2}{|c|}{ Motor properties } & \multicolumn{2}{|c|}{$\begin{array}{c}\text { Nominal } \\
\text { working point }\end{array}$} \\
\hline Parameter & Value & Parameter & Value \\
\hline$R_{\mathrm{S}}[\Omega]$ & $25.9 \cdot 10^{-3}$ & $P_{\mathrm{N}}[\mathrm{W}]$ & $87.0 \cdot 10^{3}$ \\
\hline$R_{\mathrm{R}}[\Omega]$ & $18.0 \cdot 10^{-3}$ & $\omega_{\mathrm{N}}[\mathrm{rad} / \mathrm{s}]$ & 150 \\
\hline$L_{\mu}[\mathrm{H}]$ & $27.6 \cdot 10^{-3}$ & $\Psi_{\mathrm{N}}[\mathrm{Wb}]$ & 0.84 \\
\hline$L_{\sigma}[\mathrm{H}]$ & $1.3 \cdot 10^{-3}$ & $u_{\mathrm{N}}[\mathrm{V}]$ & 230 \\
\hline$p[1]$ & 2 & $i_{\mathrm{N}}[\mathrm{A}]$ & 180 \\
\hline
\end{tabular}
original 4th order system will be reduced into a new 3rd order system. The $\Psi_{\mathrm{q}}$ component of the flux will always be zero. This will help when designing the control algorithm, because the torque of the machine will depend on the $\Psi_{\mathrm{d}}$ and $i_{\mathrm{q}}$ components only. Because we aim to design a sliding mode controller for
Table 1: Motor parameters.

the torque of the IM, we will set $\Psi_{\mathrm{d}}$ and $i_{\mathrm{q}}$ as our sliding plane, and all investigations will be related to this plane. The last variable current $i_{\mathrm{d}}$ remains uncontrolled, but it follows from the following analysis that it can be controlled vicariously by proper selection of the input voltage vector or by limiting the area of operation.

Now, let us investigate which behaviors of the IM can be expressed from the system (10).

\subsection{Reaction to voltage vector}

Before designing the controller, let us study the response of the machine to the voltage vector that is applied to it. In order to simplify the analysis, let us begin by analyzing the reaction on the four vectors in the directions of the $d, q$ axes with the amplitude of the virtual DC-link voltage, instead of the six vectors with an unknown heading angle that would be available at any moment. Fig. 6 depicts the change in the state of the machine (in the flux/current plane). The arrows with a rhombus visualize the change in the state of the machine (torque). The arrows visualize the change in the id component (right orientation means a positive sign, left orientation means a negative sign). The investigated machine [8] has the following parameters, summarized in Tab. 1.

The figures show that, for different operation states, the voltage vectors have a different effect on the machine. We can see from the rightmost column of figures, where the machine is operating at nominal speed, that the application of the voltage vector in the direction of the $d$ axis will either increase or decrease the flux, but it will always decrease the torque. As assumed, the application of the voltage vector in the negative direction of the $q$ axis will lead to a large decrease in $i_{\mathrm{q}}$ (torque), but an increase in torque is not possible because the machine has already reached its limits. 

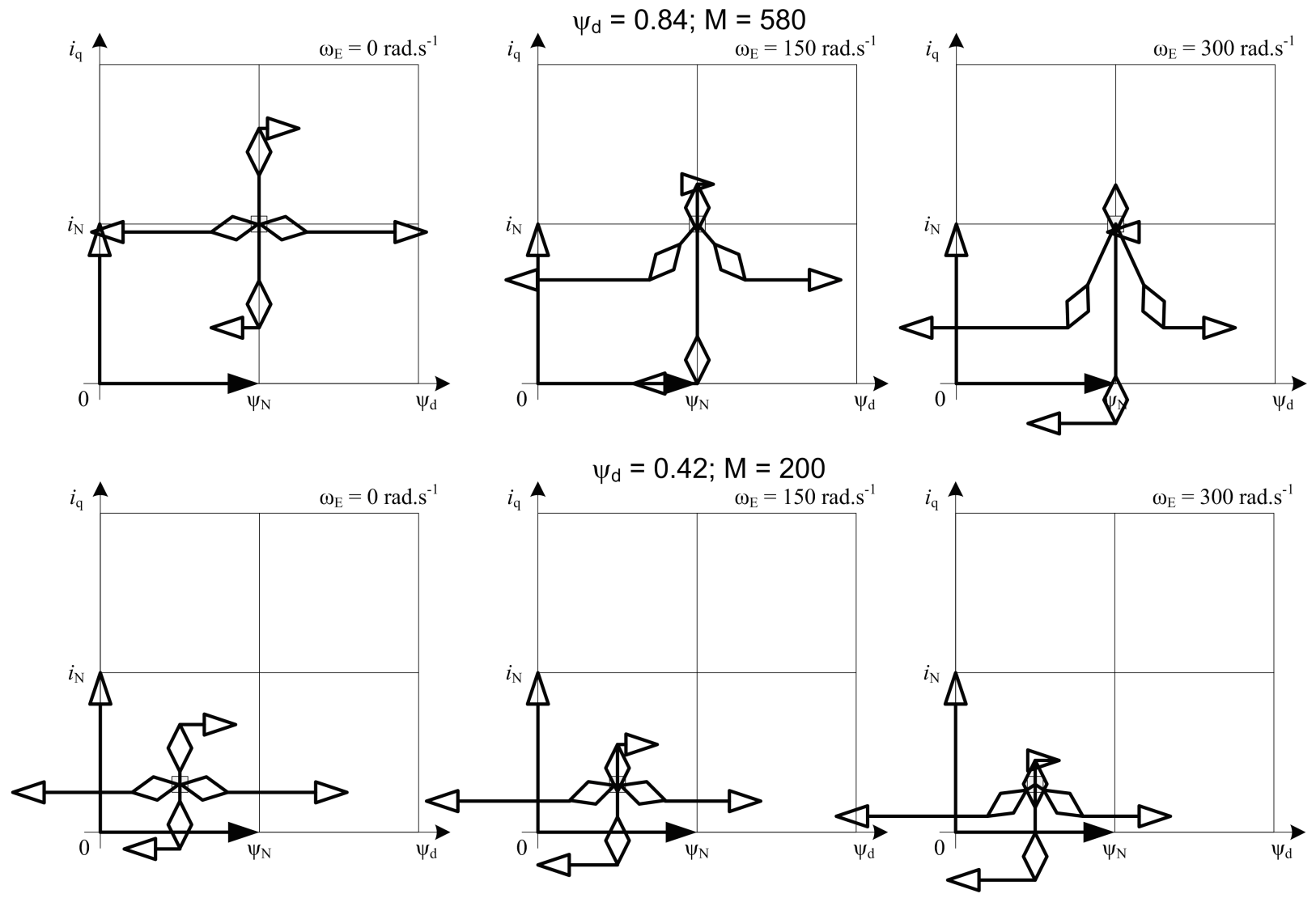

Figure 6: Reaction to applied voltage vector.

\section{Design of the controller}

We might assume that the limitation of the working area for torque control would depend on a number of factors. Let us to investigate the existence condition for sliding mode torque control.

Typically, the flux is kept constant and the torque control is actualized via changes in the orthogonal current $\left(i_{\mathrm{q}}\right)$. In this case, the sliding line is orthogonal to the flux axis. There are typically two movements: upwards to increase the torque, and downwards to decrease the torque [9]. Both cases are symbolically depicted in Fig. 7.

There is a sliding mode that increases the torque, if there is always a pair of voltage vectors, both increasing iq, where the first vector derives positive torque and the second vector derives negative torque.

Conversely, there is sliding mode that decreases the torque if there is always a pair of voltage vectors, both decreasing $i_{\mathrm{q}}$, where the first vector derives positive torque and second vector derives negative torque. Obviously, the worst case (the strongest condition for existence) subsists in a voltage vector that simultaneously increases both the flux and the orthogonal current component. Since the analysis is being conducted in the rotating plane, there is a 60 -degree uncertainty in the available direction of the voltage vector (see Fig. 8). There will always be one vector
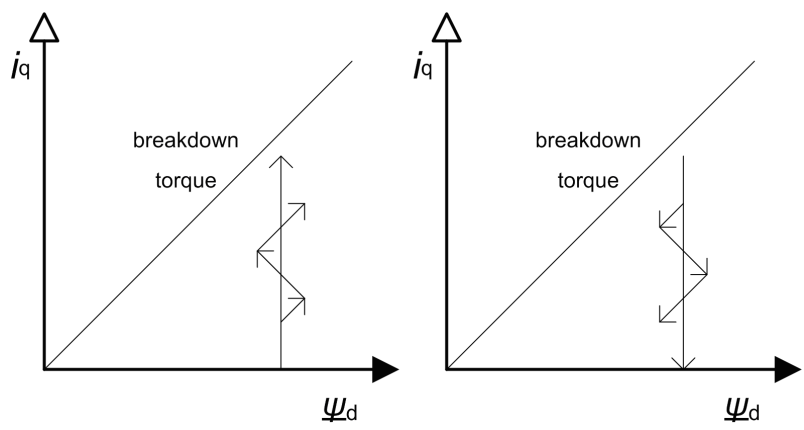

Figure 7: Principle of sliding mode torque control.

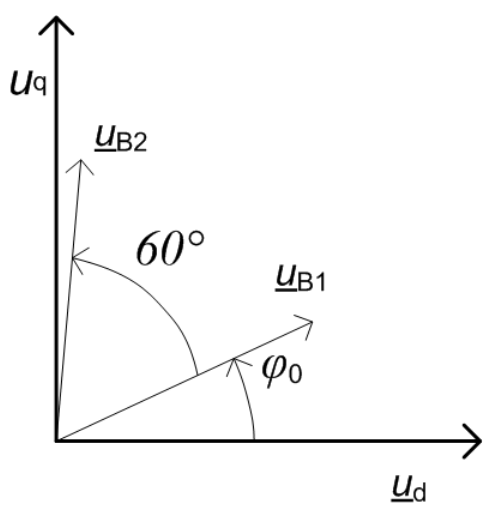

Figure 8: Principle of sliding mode torque control. 


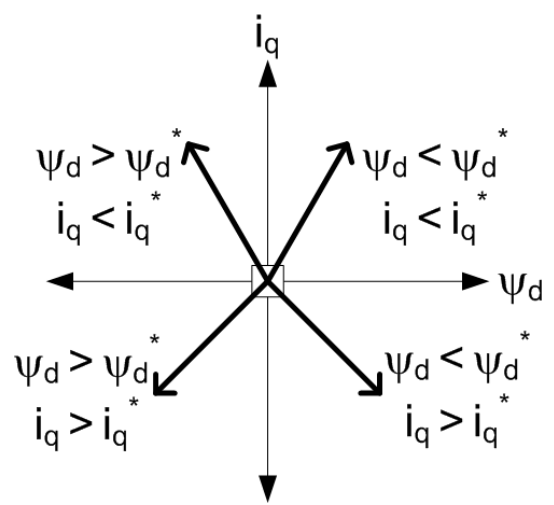

Figure 9: Principle of sliding mode torque control.

with the direction between $u_{\mathrm{B} 1}$ and $u_{\mathrm{B} 2}$, but the exact value of the angle is random. The optimization task consists in appropriately selecting the 60-degree region (choice of $\varphi_{0}$ - see the figure below) in order to extend the guaranteed working area as far as possible.

A sliding mode controller for a drive fed by a matrix converter was designed on the basis of the analysis presented here. The input for the regulation is the desired flux, $\Psi_{\mathrm{d}}^{*}$, and the desired torque, $M^{*}$. From these two values we calculate the current component $i_{\mathrm{q}}^{*}$ that is required for torque generation. The values for $\Psi_{\mathrm{d}}^{*}$ and $i_{\mathrm{q}}^{*}$ are then compared with the current values for $\Psi_{\mathrm{d}}^{*}$ and $i_{\mathrm{q}}^{*}$, and according to the result of this comparison the vector that should be generated is selected to produce the desired movement of the operation point of the machine, see Fig. 9.

The vectors that are required were selected on the basis of an analysis of the reaction to the voltage vector. It followed from the analysis that the decrease in the torque is more rapid than the increase. For this reason, we choose to boost the increase in the $i_{\mathrm{q}}$ component when an increase in torque is required by requiring a vector with an angle of 60 degrees. Conversely, we smooth out the decrease in the iq component when a decrease in torque is required, by requiring a voltage vector with an angle of -45 degrees.

Taking the uncertainty of the available voltage vectors into account: torque-increasing voltage vectors are selected randomly from the interval between 30 and 90 degrees, and torque decreasing voltage vectors belong to the interval between 15 and 75 degrees. A simplified block diagram of the controller implemented in Matlab/Simulink is depicted in Fig. 10. From the measured voltages and currents, we recalculate values that cannot be measured directly - motor flux $\Psi$, and angle of the flux phasor $\varphi$. Current values of $\Psi_{\mathrm{d}}$ and $i_{\mathrm{q}}$ are expressed on the basis of knowledge of these two variables. The most suitable voltage vector available is then selected by searching for the maxima of scalar multiplication of the output vectors of the required vector and the available converter.

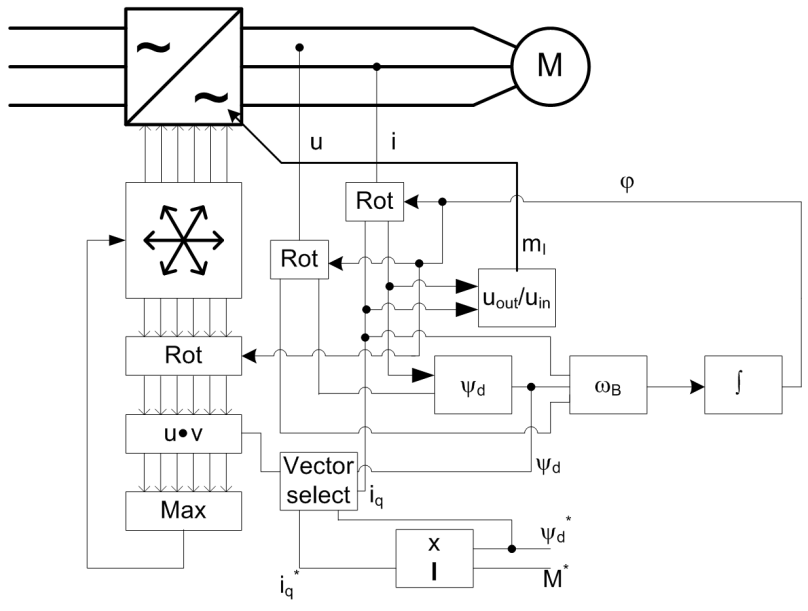

Figure 10: Controller block diagram.

\section{Simulation results}

The simulation results of this control are depicted in Fig. 11. It shows the results of the simulation with the proposed control strategy for desired values of $\Psi_{\mathrm{d}}=0.84 \mathrm{~Wb}$ and various load torques. The red line shows the desired torque and the green line represents the real torque. It can be seen that the controller tracks the desired value very rapidly, and without oscillations in the transitions.

Fig. 12 shows the trajectory of the controlled variables in the polar coordinate system. It can be seen that the flux of the machine first reaches its desired value, and then starts chattering around the desired flux line and the orthogonal current component is adjusted according to the required mechanical torque. Fig. 13 shows the selected voltage vectors. It can be seen that the voltage vectors are scattered around the desired vectors $\pm 60^{\circ}$ and $\pm 45^{\circ}$.

\section{Conclusion}

We have presented the control strategy for a matrix converter based on the virtual DC-link concept and generalized sliding mode modulation of the virtual inverter. The simulation results look very promising, and show that sliding mode control of the virtual inverter can operate simultaneously with independent input sinusoidal modulation. The responses of the controller to a change in the desired state are rapid, and there is no overshoot. However, the real impact of the modulation can only be evaluated after the algorithm has been implemented on the real converter prototype. Nevertheless, it forms a solid basis for further investigations and for improving the suggested control concept for a matrix converter induction motor drive system. An analysis of the behavior of the induction machine based on the state space description has also been presented. The output of the analysis 

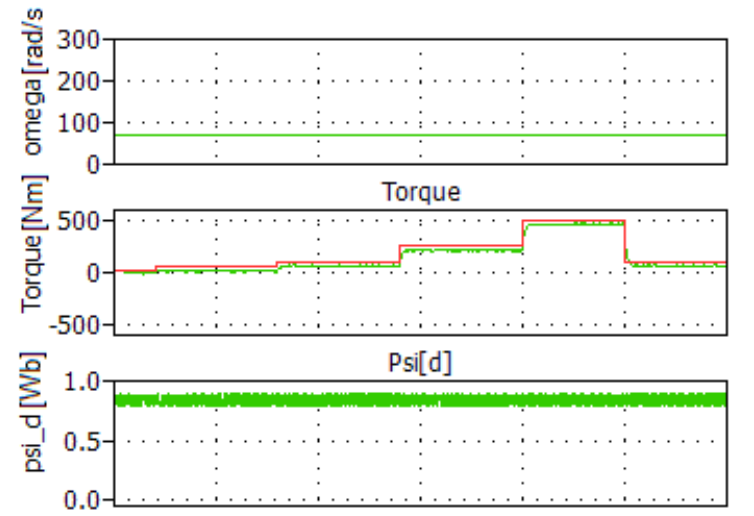

i[d]

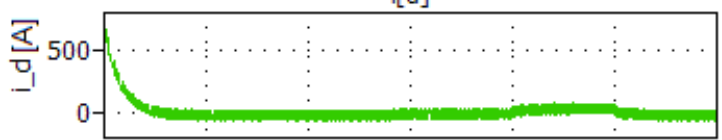

i[q]

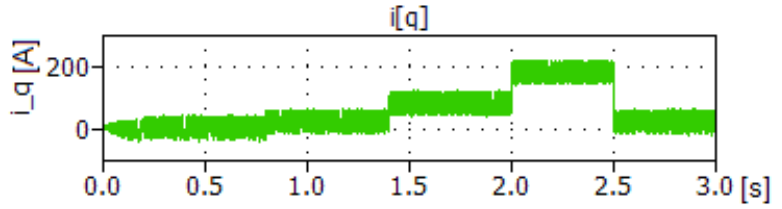

Figure 11: Reaction of the drive.

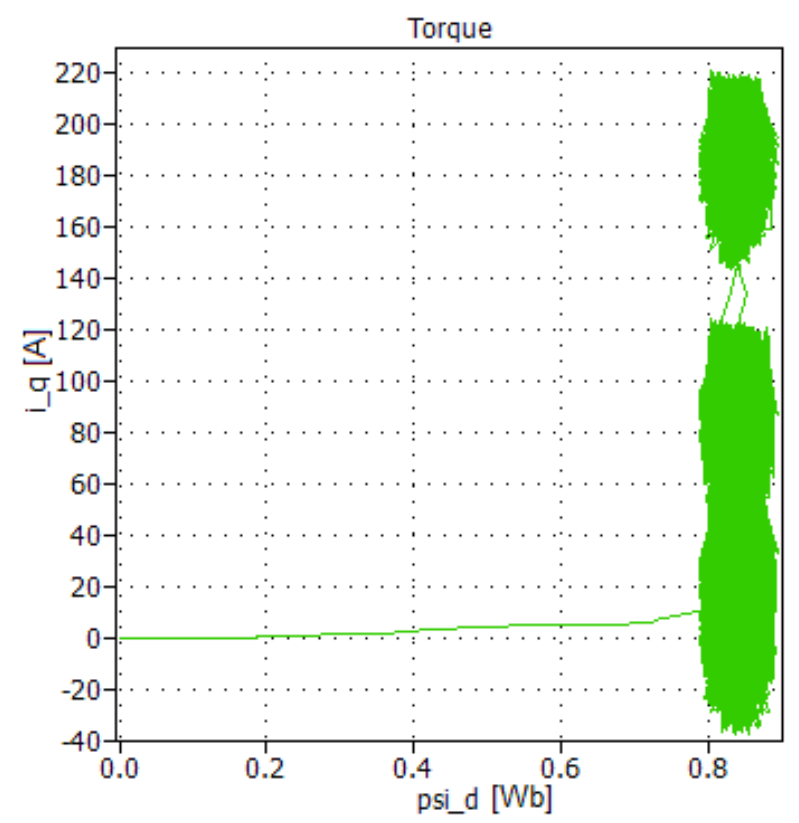

Figure 12: Trajectory of the controlled variables.

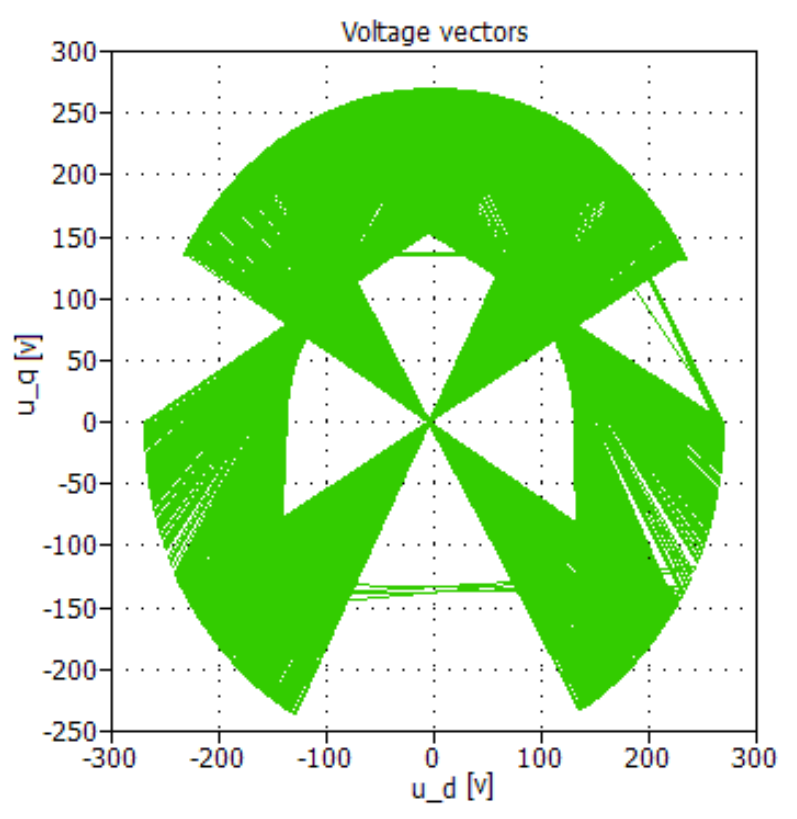

Figure 13: Generated output voltage vectors.

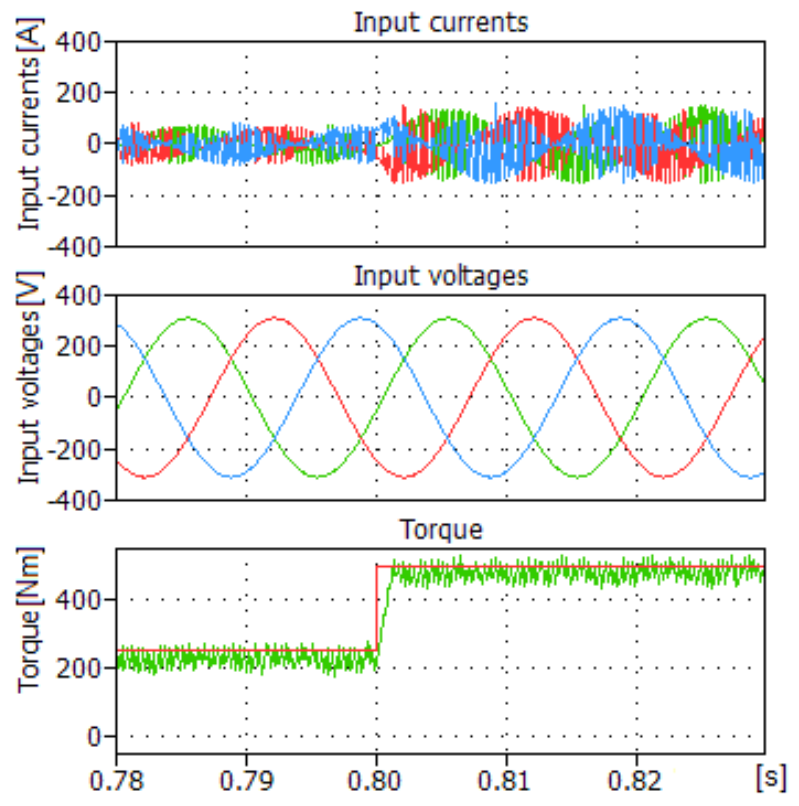

Figure 14: Input of the converter. 
shows that stable operation of the induction machine is limited by its breakdown torque, independently from the supply voltage and the rotor speed. This result is also useful for other types of converters.

\section{Acknowledgements}

The research described in this paper has been supervised by Ing. Stanislav Flígl, PhD., FEE CTU in Prague, and has been supported by the Grant Agency of the Czech Technical University in Prague, grant No. SGS12/067/OHK3/1T/13.

\section{References}

[1] P. Brandstetter, L. Hrdina and P. Simonik. Properties of selected direct torque control methods of induction motor In Industrial Electronics (ISIE), 2010 IEEE International Symposium on, pp. 1456-1461, 2010.

[2] C. Klumpner, F. Blaabjerg, I. Boldea and P. Nielsen. New modulation method for matrix converters. Industry Applications, IEEE Transactions on 42(3):797-806, 2006.

[3] J. W. Kolar, T. Friedli, J. Rodriguez and P. W. Wheeler. Review of three-phase PWM AC$\mathrm{AC}$ converter topologies. Industrial Electronics, IEEE Transactions on 58(11):4988-5006, 2011.
[4] J. Lettl. Matrix Converter Induction Motor Drive. In Proceedings of EPE-PEMC 2006 1-4:11191124. Portoroz, 2006.

[5] J. Lettl and S. Fligl. PWM strategy applied to realized matrix converter system. In Proceedings of Piers 200\%. Prague, 2007.

[6] D. W. Novotny and T. A. Lipo. Vector Control and Dynamics of $A C$ Drives. 1st edition, Oxford, Oxford University Press, 1996.

[7] S. Ryvkin. Sliding mode technique for AC drive. In Proc.10th Int. Power Electron. \& Motion Control Conf., EPE - PEMC. Dubrovnik \& Cavtat, Croatia, 2005.

[8] S. Ryvkin, R. Schmidt-Obermoeller and A. Steimel. Sliding-Mode-Based Control for a ThreeLevel Inverter Drive. IEEE Transaction on Industrial Electronics 55(11):3828-3835, 2008.

[9] V. I. Utkin, J. Gldner and J. Shi. Sliding mode control in electromechanical system. Philadelphia: Taylor \& Francis, London, 1999.

[10] P. Vas. Sensorless vector and direct torque control. Oxford, Oxford University Press, 1998. 